\title{
Personalidad e infracciones frecuentes de normas de tránsito*
}

\section{Personality and frequent violations of traffic rules}

\section{Nicolasa María Durán Palacio** \\ Norman Darío Moreno Carmona \\ Fundación Universitaria Luis Amigó. Medellín, Colombia}

Recibido: 2 de septiembre de 2015 Revisado: 3 de octubre de 2015 Aceptado: 2 de diciembre de 2015

\section{Resumen}

La Organización Mundial de la Salud (2013), alerta a los gobiernos a tomar medidas urgentes para prevenir accidentes de tránsito. Si no se intervienen, en el 2030 serán la quinta causa de muerte. Este estudio establece el perfil psicológico de conductores de vehículo infractores frecuentes de normas de tránsito, en una muestra de 295 adultos de ambos sexos. Utilizando el cuestionario factorial de personalidad 16PF-5 y una escala de creencias, conocimientos, y comportamientos sobre seguridad vial, se identificaron relaciones significativas entre conducta infractora y creencias, además de los factores de personalidad y variables específicas que predicen la propensión a las infracciones frecuentes de normas de tránsito.

Palabras clave: personalidad, comportamiento social, normas de tránsito, infracciones. 


\section{Abstract}

The World Health Organization (2013) has alerted governments to take urgent measures to prevent traffic accidents. If no intervention is made, they will be the fifth leading cause of death in 2030. This study established the psychological profile of frequent offenders of traffic rules in a sample of 295 drivers of both sexes. Using the 16PF-5 Personality Questionnaire and a scale of beliefs, knowledge, and road safety behaviors, significant relationships between offending behavior and beliefs were identified, in addition to the specific personality factors and variables that predict susceptibility to frequent violations of traffic rules.

Keywords: Personality, behavior social, traffic rules, infringements.

\section{Introducción}

Conforme el hombre ha modernizado y aumentado sus medios de transporte terrestre, los riesgos y accidentalidad también se elevan notoriamente. Aunque los países han implementado progresivamente distintas reglamentaciones, campañas publicitarias educativas y técnicas pedagógicas de tránsito y transporte, desde el año 2007 las lesiones, traumas y muertes por accidentes de tránsito son significativamente alarmantes.

El último informe de la Organización Mundial de la Salud reportó 1,24 millones de muertes anuales en el mundo por incidentes de tráfico en vías públicas. Los países con ingresos medios presentaron las mayores tasas de mortalidad. En las naciones de las Américas las defunciones alcanzaron el 42 $\%$, mientras que en los países con ingresos altos la proporción fue de $23 \%$. Peatones, ciclistas y motociclistas son el grupo humano más vulnerables en las vías (OMS, 2013).

En Colombia en el año 2014, el Instituto Nacional de Medicina Legal documentó 50.574 casos atendidos por accidentes de transportes durante el año. 6.402 personas fallecieron por esta causa $(12,66 \%)$ y hubo 44.172 lesionados $(87,34 \%)$. Las muertes aumentaron $2,94 \%$ y los lesionados en $5,62 \%$ respecto al año 2013 . Antioquia es uno de los cinco departamentos más afectados por muertes y lesiones en accidentes de tránsito con una tasa de 15,47 \% fallecidos (987), y 85,06 \% lesiona- dos (5.425) por cada 100.000 habitantes. De estas cifras, Medellín aporta el 11,88 \% de las muertes (290) y 2.965 de los lesionados. El exceso de velocidad y la violación a las normas de tránsito fueron las causas más relevantes (Vargas, 2015).

Aunque las sanciones al comportamiento infractor de las normas de tránsito son elementos importantes para la regulación de la convivencia y uso de las vías públicas, es necesario reconocer los aspectos psicológicos involucrados en la trama relacional que establecen los distintos actores viales, ya que la mayoría de los siniestros son, en gran medida, el resultado de un proceso decisional de quien conduce.

Varios estudios en psicología del tráfico han utilizado instrumentos de exploración de la personalidad (Ponce, 2015; Aniței, Chraif, Burtăverde y Mihăilă, 2014; Poó, Ledesma y Montes, 2008; Sümer, 2003), estilos cognitivos (Parker et al., 1992; Elander et al., 1993; Hansen, 2005) perceptuales (Jamenson, McLelan y Jackson, 1971; Summala y Näätänen, 1974; Wilde, 1982; Summala, 1988) y del comportamiento (Stokols et al., 1978; Knapper y Cropley, 1981; Reason et al., 1990), al analizar las influencias de las variables personales en la conducción.

En la revisión de antecedentes para este trabajo, no se halló evidencia sobre el uso del Cuestionario de Personalidad 16PF-5 (Catell, Catell y Catell, 1993), aunque sí el Big Five (Caprara, Barbaranelli 
y Borgogni, 1993) para la explicación de los factores de personalidad implicados en la accidentalidad vial.

Taubman-Ben-Ari, Mikulincer y Gillath (2004, 2012) hallaron una relación altamente significativa entre estilos de conducción y personalidad. Estos autores diseñaron el Inventario Multidimensional de Estilos de Conducción (MDSI) que permite evaluar el estilo de conducción a través de siete dimensiones: riesgo, agresión, disociación, ansiedad, prudencia, cordialidad y estrés. La agresión, la conducción riesgosa o temeraria y la búsqueda de sensaciones en los conductores hombres, constituyó el patrón diferencial predictor de riesgo, mientras que en las mujeres, la agresión, la cordialidad y el estrés fueron los predictores característicos de su estilo de conducción.

Aniței, Chraif, Burtăverde y Mihăilă (2014), en un estudio con 100 participantes jóvenes conductores de Rumania -país que ocupó el primer lugar en número de muertes por accidentes de tránsito en Europa-, utilizando el cuestionario de personalidad Big Five, encontraron que la baja estabilidad emocional, afabilidad y autoconciencia, así como la alta extraversión y apertura a la experiencia, predicen el comportamiento de conducción agresiva.

En España, Alonso, Esteban, Calatayud, Medina, Montoro y Egido (2003), insisten en que la investigación del factor humano en la seguridad vial es fundamental para orientar la intervención en la prevención del accidente y sus causas, además de la utilidad del conocimiento psicológico -derivado de investigaciones- en la evaluación de conductores para el otorgamiento de las licencias y permisos para conducir.

En Argentina, Ledesma, Montes, Poó y López-Ramón (2009), al estudiar la falta de atención del conductor y las variables psicológicas relacionadas con el fenómeno de la conducción, concluyeron que las diferencias individuales en la falta de atención del conductor involucrado en accidentes de tránsito, son relevantes en los estudios de la siniestralidad vial. Aplicando la escala de errores de conducción y atención relacionados (ARDES), hallaron que los fallos en la conducción están fuertemente asociados con la propensión general al error, la falta de atención al realizar las actividades cotidianas y los rasgos de personalidad disociativos.

Posteriormente, Montes, Poó, Valle y López-Ramón (2012, 2014), en estudio comparativo analizaron la relación entre las tendencias disociativas e incidentes de tránsito en conductores de la ciudad de Mar del Plata, Argentina, aplicando la versión modificada de la Escala de Experiencias Disociativas (DES) de Bernstein y Putman (1986), advirtieron que los sujetos que participaron en algún tipo de accidente de tránsito puntuaron más alto en la escala de experiencias disociativas y sus tres subescalas: amnesia, absorción y despersonalización.

La investigación de Norza, Granados, Useche, Romero y Moreno (2014) sobre los componentes descriptivos y explicativos de la accidentalidad vial en el territorio colombiano, realizada en 38 seccionales de tránsito y transporte de la Policía Nacional, encontró que los conductores con estilos de conducción iracundo, ansioso, riesgoso y de alta velocidad son los que cometen más infracciones y accidentes en el país. El desacato a las normas de tránsito es la principal causa de accidentalidad. La agresividad, hostilidad y estrés en el tráfico son factores que aumentan la probabilidad de incidentes. Las campañas de prevención no son captadas por la población más involucrada en los accidentes.

\section{Método}

Estudio ex post facto retrospectivo con dos grupos, uno de ellos cuasi control (Montero y León, 2007). Diseño muestral no probabilístico que incluyó 295 conductores, mayores de edad y de ambos sexos. $72,5 \%$ hombres y $27,5 \%$ mujeres, asistentes a los cursos pedagógicos que los infractores deben realizar si desean un descuento de ley en la multa por alguna transgresión de tránsito. Estos sujetos aceptaron participar voluntariamente en el estudio y luego de firmar un consentimiento informado. Al revisar la base de datos del SIMIT, se encontró que 141 sujetos correspondían a infractores no frecuentes ( $47.8 \%$ ), mientras que 154 fueron clasificados como infractores frecuentes $(52.2 \%)$. 
La tipificación del comportamiento infractor de las normas de tránsito se estableció de acuerdo a la disposición del Ministerio de Transporte y al Código Nacional de Tránsito, Ley 769 de 2002. Un infractor es aquel actor del tránsito declarado responsable por las autoridades respectivas de infringir o transgredir una norma de tránsito, y a quien habiendo sido citado para comparecer, cancela el valor de la multa (Superintendencia de Puertos y Transporte. Ministerio de Transporte, 2010).

En este estudio se define al infractor frecuente como aquel conductor que cometió la misma infracción al menos dos veces en el último año o cometió tres o más infracciones diferentes en los últimos dos años. Las infracciones que se tuvieron en cuenta para esta clasificación fueron aquellas consideradas de mayor riesgo para la seguridad vial.

Instrumentos: se utilizó el Cuestionario de Personalidad 16PF-5 (Catell, Catell y Catell 1993), que evalúa 16 factores básicos de la personalidad y proporciona 4 factores secundarios que representan un resumen breve del perfil del sujeto. Para obtener información más completa se elaboró una escala de actitudes, validada por expertos, hacia la movilidad y la seguridad vial, conformada por tres subescalas: creencias, conocimientos y comportamientos.

- 16PF-A Frío, impersonal y distante - afable, cálido, generoso y atento

- 16PF-B Pensamiento concreto - pensamiento abstracto

- 16PF-C Reactivo, emocionalmente inestable emocionalmente estable, adaptable y maduro

- 16PF-E Deferente y cooperativo, que evita conflictos - dominante, fuerte y asertivo

- 16PF-F Serio, cohibido y solícito - animado y espontáneo

- 16PF-G Muy suyo e inconformista - atento a las normas y cumplidor

- 16PF-H Asustadizo, apocado y tímido - atrevido, emprendedor y arriesgado
- 16PF-I Objetivo y poco sentimental - sensible, esteta y sentimental

- 16PF-L Confiado, no suspicaz y acogedor - vigilante, suspicaz, escéptico y cauteloso

- 16PF-M Realista, práctico y orientado a la acción - abstracto, imaginativo y orientado a las ideas

- 16PF-N Abierto, espontáneo y sencillo - receloso, discreto y cerrado

- 16PF-0 Seguro, despreocupado y satisfecho aprensivo, dubitativo y preocupado

- 16PF-Q1 Tradicional y apegado a lo familiar abierto al cambio y experimental

- 16PF-Q2 Integrado en el grupo y afiliativo - seguro, solitario e individualista

- 16PF-Q3 Tolerante con el desorden, condescendiente y flexible - perfeccionista, organizado y autodisciplinado

- 16PF-Q4 Relajado, plácido y placiente - tenso, enérgico, impaciente y presionado

Manipulación de Imagen: Esfuerzo por dar mala imagen, problemas de autoestima - esfuerzo por causar buena impresión

Infrecuencia: oposicionismo al responder el cuestionario. Tendencia a responder $\mathrm{B}$ (indeciso)

Aquiescencia: identificar aquellos estilos de respuesta en que el examinado contesta verdadero, independiente del contenido

Extraversión: introvertido y socialmente inhibido - extrovertido y socialmente participativo

Ansiedad: poco ansioso e imperturbable - muy ansioso e intranquilo

Dureza: de mentalidad abierta, receptivo e intuitivo - de mentalidad dura, resolutivo e insensible

Independencia: acomodaticio, conformista y desinteresado - independiente, persuasivo y voluntarioso

Autocontrol: incontrolado y obedece a sus impulsos - autocontrolado y que inhibe sus impulsos 
Si bien el Cuestionario de Personalidad 16PF-5 no ha sido utilizado en estudios con infractores de normas de tráfico y transporte, en esta investigación se optó por su uso, reconociendo su capacidad de predicción de rasgos y atributos conductuales en situaciones en que las personas son afectadas por estímulos y convenciones sociales.

Procedimiento: se evaluó la capacidad de discriminación de los reactivos por subescalas, utilizando la prueba de Levene y $\mathrm{T}$ - Student para muestras independientes, y el Alpha de Cronbach para analizar la confiabilidad en las escalas de creencias y comportamientos, tipo Likert. Se realizó un análisis del índice de dificultad para la escala de conocimientos.

El análisis condujo a un instrumento compuesto por 14 reactivos de creencias $(\alpha=, 870), 11$ de comportamiento $(\alpha=, 773)$ y 10 reactivos de co- nocimiento. Los reactivos de las tres escalas presentaron un adecuado índice de discriminación $(\mathrm{p} \leq, 01)$ y un aceptable índice de dificultad en la de conocimiento.

\section{Resultados}

En la composición de la muestra, la mayor parte de los conductores encuestados son infractores frecuentes (tabla 1). En alto porcentaje hombres, con más de 35 años, edad media de 41,4 años y una $D E=13,8$. La prueba Chi-cuadrado $\left(\chi^{2}=8,686\right)$ indica una diferencia estadísticamente significativa entre los sexos $(p \leq, 01)$. Los varones presentaron una tendencia más alta a ser infractor frecuente de normas de tránsito que las mujeres. A medida que aumenta la edad crece la propensión a cometer infracciones. La población mayor a 35 años se ubica en mayor proporción como infractores habituales.

Tabla 1.

Características demográficas de los participantes por grupos poblacionales.

\begin{tabular}{|c|c|c|c|c|c|c|}
\hline \multirow[t]{2}{*}{ Característica } & \multicolumn{2}{|c|}{$\begin{array}{l}\text { Total muestra } \\
\qquad(\mathrm{N}=295)\end{array}$} & \multicolumn{2}{|c|}{$\begin{array}{l}\text { No infractores frecuentes } \\
\qquad(\mathrm{N}=141)\end{array}$} & \multicolumn{2}{|c|}{$\begin{array}{l}\text { Infractores frecuente } \\
\qquad(\mathrm{N}=154)\end{array}$} \\
\hline & $\mathrm{n}$ & $\%$ & $\mathrm{n}$ & $\%$ & $\mathrm{n}$ & $\%$ \\
\hline \multicolumn{7}{|l|}{ Sexo } \\
\hline Hombres & 214 & 72,5 & 91 & 64,5 & 123 & 79,9 \\
\hline Mujeres & 81 & 27,5 & 50 & 35,5 & 31 & 20,1 \\
\hline \multicolumn{7}{|l|}{ Edad } \\
\hline $17-25$ & 44 & 14,9 & 25 & 17,7 & 19 & 12,3 \\
\hline $26-35$ & 67 & 22,7 & 35 & 24,8 & 32 & 20,8 \\
\hline $36-50$ & 108 & 36,6 & 49 & 34,8 & 59 & 38,3 \\
\hline $51-80$ & 76 & 25,7 & 32 & 22,7 & 44 & 28,5 \\
\hline \multicolumn{7}{|l|}{ Escolaridad } \\
\hline No estudió & 18 & 6,1 & 6 & 4,3 & 12 & 7,8 \\
\hline Primaria incompleta & 1 & 0,3 & 0 & 0 & 1 & 0,6 \\
\hline $5^{\circ}$ & 8 & 2,7 & 4 & 2,8 & 4 & 2,6 \\
\hline Secundaria incompleta & 12 & 4,1 & 7 & 5,0 & 5 & 3,2 \\
\hline $11^{\circ}$ & 53 & 18,0 & 22 & 15,6 & 31 & 20,1 \\
\hline Técnico o tecnólogo & 72 & 24.4 & 36 & 25,5 & 36 & 23,4 \\
\hline Pregrado & 69 & 23,4 & 35 & 24,8 & 34 & 22,1 \\
\hline Posgrado & 62 & 21,0 & 31 & 22,0 & 31 & 20,1 \\
\hline \multicolumn{7}{|l|}{ Aprendió a conducir } \\
\hline Escuela de conducción & 147 & 49,8 & 76 & 53,9 & 71 & 46,1 \\
\hline
\end{tabular}




\begin{tabular}{|c|c|c|c|c|c|c|}
\hline \multirow[t]{2}{*}{ Característica } & \multicolumn{2}{|c|}{$\begin{array}{l}\text { Total muestra } \\
\qquad(\mathrm{N}=295)\end{array}$} & \multicolumn{2}{|c|}{$\begin{array}{l}\text { No infractores frecuentes } \\
\qquad(\mathrm{N}=141)\end{array}$} & \multicolumn{2}{|c|}{$\begin{array}{l}\text { Infractores frecuentes } \\
\qquad(\mathrm{N}=154)\end{array}$} \\
\hline & $\mathrm{n}$ & $\%$ & $\mathrm{n}$ & $\%$ & $\mathrm{n}$ & $\%$ \\
\hline Familiar o amigo & 115 & 39,0 & 51 & 36,2 & 64 & 41,6 \\
\hline Solo & 33 & 11,2 & 14 & 9,9 & 19 & 12,3 \\
\hline \multicolumn{7}{|l|}{ Obtuvo la licencia } \\
\hline Escuela de conducción & 211 & 72,3 & 107 & 76,4 & 104 & 68,4 \\
\hline Tramitador & 81 & 27,7 & 33 & 23,6 & 48 & 31,6 \\
\hline \multicolumn{7}{|l|}{ Tipo de vehículo } \\
\hline Moto & 62 & 21,0 & 29 & 20,6 & 33 & 21,4 \\
\hline Carro particular & 203 & 68,8 & 102 & 72,3 & 101 & 65,6 \\
\hline Taxi & 18 & 6,1 & 7 & 5,0 & 11 & 7,1 \\
\hline Vehículo de carga & 11 & 3,7 & 3 & 2,1 & 8 & 5,2 \\
\hline Bus & 1 & 0,3 & 0 & 0 & 1 & 0,6 \\
\hline
\end{tabular}

Edad $M=41,4 \mathrm{DE}=13,8 \mathrm{M}$ Edad $\mathrm{NIF}=40,1 \mathrm{DE}=14,3 \mathrm{M}$ Edad $\mathrm{IF}=42,63 \mathrm{DE}=13,2$.

La mayoría de los participantes tienen educación superior, conducen vehículo particular, obtuvieron su licencia y aprendieron a manejar en escuelas de conducción. En menor proporción motociclistas. Los resultados muestran que las contravenciones frecuentes de normas de tránsito son cometidas por conductores bachilleres, con formación técnica, profesional y posgrado.

Los resultados muestran una tendencia a incrementar la frecuencia de infracciones de normas tránsito en aquellos conductores que aprendieron a conducir con un familiar, un amigo o solos, y que obtuvieron la licencia con un tramitador, sin embargo, la prueba Chi cuadrado indica que el aprender a manejar en una escuela de conducción, no representa una diferencia significativa $\left(\chi^{2}=1,790\right.$ y $\chi^{2}=2,331$ respectivamente, $\left.p \geq, 05\right)$.

La mayoría de los evaluados conducen vehículo particular y motocicleta, sin embargo no hay diferencias en el tipo de automotor entre quienes son infractores habituales y los que no son. La mayor parte de los conductores de transporte público y vehículos de carga, poco representativos en la muestra, son infractores frecuentes.

Para analizar la existencia de relación entre los distintos componentes de la actitud, se realizó una correlación de Pearson. En la tabla 2 se observa que en ambos grupos de conductores existe correlación significativa entre creencias y comportamientos $(p \leq 0,001)$. La conducta al conducir está relacionada más con las creencias que con el conocimiento de la normatividad.

Tabla 2.

Correlaciones, medias y desviaciones estándar para las puntuaciones en las subescalas de actitudes frente a la conducción según tipo de conductor.

\begin{tabular}{lcccc}
\hline Medida & $\begin{array}{c}\text { Compor- } \\
\text { tamiento. }\end{array}$ & $\begin{array}{c}\text { Conoci- } \\
\text { miento. }\end{array}$ & M & DE \\
\hline $\begin{array}{l}\text { Total } \\
\text { muestra }\end{array}$ & & & \\
\hline Creencias &, $366^{* * *}$ &,- 022 & 1,652 &, 566 \\
\hline Comportam &, 050 & 1,867 &, 550 \\
\hline Conocim & & 2,07 &, 996 \\
\hline No infractFrec &, $333^{* * *}$ &, 042 & 1,667 &, 567 \\
\hline Creencias &, 121 & 1,845 &, 541 \\
\hline Comportam & & 2,11 &, 983 \\
\hline Conocim & & & \\
\hline InfractFrecuent &, $397^{* * *}$ &,- 081 & 1,637 &, 566 \\
\hline Creencias &,- 009 & 1,887 &, 559 \\
\hline Comportam & & 2,04 & 1,01 \\
\hline Conocim & & & \\
\hline
\end{tabular}

${ }^{*} p \leq 0,05^{* *} p \leq 0,01{ }^{* * *} p \leq 0,001$. 
Los resultados del 16PF-5 (tabla 3), evidencian las frecuencias de la distribución de la muestra según su ubicación en los polos críticos de cada rasgo (puntajes PB por debajo de 3,5 o PA por encima de 7,5).

Tabla 3.

Resultados clínicos del 16PF5 por grupos de conductores.

\begin{tabular}{|c|c|c|c|c|c|c|c|c|c|c|}
\hline \multicolumn{5}{|c|}{ NO INFRACTORES FRECUENTES (141) } & \multirow[t]{2}{*}{$\begin{array}{l}\text { Factor } \\
\text { 16PF5 }\end{array}$} & \multicolumn{5}{|c|}{ INFRACTORES FRECUENTES (154) } \\
\hline PB & $\%$ & $\%$ neutros & PA & $\%$ & & PB & $\%$ & $\%$ neutros & PA & $\%$ \\
\hline 19 & 13,5 & 68,1 & 26 & 18,4 & A & 27 & 17,5 & 68,2 & 22 & 14,3 \\
\hline 16 & 11,3 & 68,1 & 29 & 20,6 & B & 28 & 18,2 & 67,5 & 22 & 14,3 \\
\hline 28 & 19,9 & 70,2 & 14 & 9,9 & $\mathrm{C}$ & 44 & 28,6 & 64,3 & 11 & 7,1 \\
\hline 11 & 7,8 & 80,9 & 16 & 11,3 & $\mathrm{E}$ & 30 & 19,5 & 72,7 & 12 & 7,8 \\
\hline 10 & 7,1 & 69,5 & 33 & 23,4 & $\mathrm{~F}$ & 18 & 11,7 & 73,4 & 23 & 14,9 \\
\hline 25 & 17,7 & 61,0 & 30 & 21,3 & $G$ & 30 & 19,5 & 67,5 & 20 & 13,0 \\
\hline 18 & 12,8 & 70,2 & 24 & 17,0 & $\mathrm{H}$ & 37 & 24,0 & 63,0 & 20 & 13,0 \\
\hline 15 & 10,6 & 73,8 & 22 & 15,6 & 1 & 21 & 13,6 & 74,0 & 19 & 12,3 \\
\hline 1 & 0,7 & 57,4 & 59 & 41,8 & $\mathrm{~L}$ & 6 & 3,9 & 40,9 & 85 & 55,2 \\
\hline 8 & 5,7 & 47,5 & 66 & 46,8 & $M$ & 5 & 3,2 & 33,8 & 97 & 63,0 \\
\hline 8 & 5,7 & 73,0 & 30 & 21,3 & $\mathrm{~N}$ & 3 & 1,9 & 63,0 & 54 & 35,1 \\
\hline 7 & 5,0 & 61,7 & 47 & 33,3 & 0 & 14 & 9,1 & 48,7 & 65 & 42,2 \\
\hline 11 & 7,8 & 62,4 & 42 & 29,8 & Q1 & 17 & 11,0 & 64,3 & 38 & 24,7 \\
\hline 0 & 0 & 43,3 & 80 & 56,7 & Q2 & 0 & 0 & 32,5 & 104 & 67,5 \\
\hline 40 & 28,4 & 54,6 & 24 & 17,0 & Q3 & 45 & 29,2 & 57,1 & 21 & 13,6 \\
\hline 6 & 4,3 & 45,4 & 71 & 50,4 & $\mathrm{Q} 4$ & 3 & 1,9 & 41,6 & 87 & 56,5 \\
\hline 30 & 21,3 & 63,8 & 21 & 14,9 & $\mathrm{MI}$ & 47 & 30,5 & 60,4 & 14 & 9,1 \\
\hline 0 & 0 & 40,4 & 84 & 59,6 & $\mathrm{AQ}$ & 0 & 0 & 42,2 & 89 & 57,8 \\
\hline 22 & 15,6 & 48,9 & 50 & 35,5 & IN & 28 & 18,2 & 41,6 & 62 & 40,3 \\
\hline 30 & 21,3 & 64,5 & 20 & 14,2 & EXT & 51 & 33,1 & 57,8 & 14 & 9,1 \\
\hline 14 & 9,9 & 54,6 & 50 & 35,5 & ANS & 11 & 7,1 & 51,9 & 63 & 40,9 \\
\hline 18 & 12,8 & 58,2 & 41 & 29,1 & DUR & 12 & 7,8 & 56,5 & 55 & 35,7 \\
\hline 12 & 8,5 & 67,4 & 34 & 24,1 & IND & 21 & 13,6 & 63,6 & 35 & 22,7 \\
\hline 38 & 27,0 & 48,2 & 35 & 24,8 & AUC & 42 & 27,3 & 57,1 & 24 & 15,6 \\
\hline
\end{tabular}

$\mathrm{PB}=$ Polo Bajo $\mathrm{PA}=$ Polo Alto.
En la prueba T de Student se encontraron diferencias estadísticamente significativas en los factores C, E, G, H, M, N, Q2, Q4, manipulación de imagen y extraversión (tabla 4). 
Tabla 4.

Medias, desviaciones estándar y prueba T de muestras independientes para factores de personalidad (16PF5) según tipo de conductor.

\begin{tabular}{|c|c|c|c|c|c|}
\hline \multirow[t]{2}{*}{ Factor } & \multicolumn{2}{|c|}{$\begin{array}{l}\text { No infractores frecuentes } \\
\qquad(\mathrm{N}=141)\end{array}$} & \multicolumn{2}{|c|}{$\begin{array}{l}\text { Infractores frecuentes } \\
\qquad(\mathrm{N}=154)\end{array}$} & \multirow[b]{2}{*}{$\mathrm{t}$} \\
\hline & $M$ & $\mathrm{DE}$ & $M$ & $\mathrm{DE}$ & \\
\hline 16PF-A & 5,14 & 1,66 & 4,99 & 1,55 & ,829 \\
\hline 16PF-B & 5,23 & 1,74 & 4,85 & 1,71 & 1,871 \\
\hline 16PF-C & 4,80 & 1,53 & 4,37 & 1,62 & $2,347^{*}$ \\
\hline 16PF-E & 5,20 & 1,28 & 4,79 & 1,45 & $2,541^{*}$ \\
\hline 16PF-F & 5,39 & 1,62 & 5,16 & 1,69 & 1,215 \\
\hline 16PF-G & 5,42 & 1,91 & 4,93 & 1,60 & $2,400^{*}$ \\
\hline 16PF-H & 5,24 & 1,60 & 4,77 & 1,68 & $2,452^{*}$ \\
\hline 16PF-I & 5,55 & 1,56 & 5,31 & 1,61 & 1,343 \\
\hline 16PF-L & 6,38 & 1,41 & 6,69 & 1,61 & $-1,761$ \\
\hline 16PF-M & 6,45 & 1,47 & 6,97 & 1,52 & $-2,946^{* *}$ \\
\hline $16 \mathrm{PF}-\mathrm{N}$ & 5,60 & 1,39 & 6,11 & 1,47 & $-3,083^{* *}$ \\
\hline 16PF-O & 5,91 & 1,48 & 6,10 & 1,70 & $-1,014$ \\
\hline 16PF-Q1 & 5,60 & 1,61 & 5,51 & 1,63 & ,472 \\
\hline 16PF-Q2 & 6,74 & 1,36 & 7,09 & 1,57 & $-2,057^{*}$ \\
\hline 16PF-Q3 & 4,67 & 1,80 & 4,60 & 1,78 & ,335 \\
\hline 16PF-Q4 & 6,54 & 1,56 & 6,94 & 1,49 & $-2,230^{*}$ \\
\hline Manipulación de imagen & 4,98 & 1,82 & 4,51 & 1,80 & $2,239^{*}$ \\
\hline Infrecuencia & 7,21 & 1,67 & 7,32 & 1,78 &,- 592 \\
\hline Aquiescencia & 5,75 & 2,10 & 5,90 & 2,30 &,- 587 \\
\hline Extraversión & 4,81 & 1,76 & 4,33 & 1,83 & $2,274^{*}$ \\
\hline Ansiedad & 5,97 & 1,92 & 6,29 & 1,98 & $-1,409$ \\
\hline Dureza & 5,58 & 1,86 & 5,99 & 1,85 & $-1,875$ \\
\hline Independencia & 5,51 & 1,61 & 5,34 & 1,66 & ,907 \\
\hline Autocontrol & 4,97 & 2,12 & 4,71 & 1,94 & 1,089 \\
\hline
\end{tabular}

${ }^{*} p \leq 0,05^{* *} p \leq 0,01 * * * p \leq 0,001$.

El 28,6\% de los infractores frecuentes (tabla 3) obtuvo puntajes en el polo bajo del factor $C$ (estabilidad emocional), indicando tendencias a mostrarse reactivo y emocionalmente inestable. La media general del grupo (tabla 4) presenta mayor propensión hacia el polo bajo que los no infractores frecuentes.

En la tabla 4, el 19,5\% de los infractores frecuentes puntuó hacia el polo bajo en el factor $\mathrm{E}$ (asertividad), tendiendo a ser deferentes y a evitar conflictos para no tener que asumir y reconocer errores. La media del grupo evidencia la predis- posición opuesta del grupo no infractor frecuente. De acuerdo con Catell, Catell y Catell (1993), menores puntajes en el factor $E$ indican que los sujetos no reconocen ni asumen su ira, de modo que no están preparados para manifestarla o controlarla, asunto que al vincularse con la actividad de la conducción predice la probabilidad de reacción explosiva e incidencia en infracciones y accidentes.

En el factor $G$ (atención a las normas), el 19,5\% de los infractores frecuentes obtuvo puntajes en el polo bajo (tabla 3), describiéndolos como in- 
conformista con las normas sociales y poco aprecio por ellas, inclinación a disimular sus errores, justificar sus conductas, toda vez que su comportamiento se desvía de la normatividad. En este caso, también la media del grupo evidencia la tendencia opuesta del conjunto no infractor frecuente (tabla 4).

Con tendencia opuesta al grupo control (tabla 3), el $24 \%$ de los infractores frecuentes obtuvo puntajes en el polo bajo del factor $\mathrm{H}$ (atrevimiento), indicando predisposición a ser asustadizos, apocados y tímidos. La prueba T de Student confirma que existen diferencias significativas entre los dos grupos (tabla 4).

La obtención de bajos valores en $\mathrm{H}$ muestra la propensión de los infractores habituales a la timidez, retraimiento social y poca afabilidad en las interacciones públicas. Relación directa con las puntuaciones menores en la dimensión $\mathrm{E}$ (extraversión), que caracteriza a las personas como propensas a la poca sociabilidad, introversión e inhibición social, indicadores de posibles dificultades de adaptación o ajuste a las normas sociales.
Los resultados en el factor $M$ (abstracción) son particularmente diferenciadores. El $63 \%$ de los infractores habituales se ubicó en el polo alto del factor (tabla 3), tipificándolos como abstractos, orientados hacia las ideas, falta de sentido práctico y con pensamientos fantasiosos.

La correlación entre C y G (tabla 5) confirma el escaso interés en los infractores frecuentes por dar una buena imagen de sí y dificultad para reconocer sus conductas problemáticas. Las puntuaciones altas de los infractores frecuentes en impracticabilidad $(M+)$, unidas a los bajos valores en atención a las normas (G-), implican dificultades en estos sujetos para la atención sostenida en las rutinas repetitivas en la conducción de vehículos.

En el factor $\mathrm{N}$ (privacidad), el 35,1\% de los infractores frecuentes se ubicó en el polo alto (tabla 3) y el grupo obtuvo una media más alta en el test respecto al grupo control (tabla 4), caracterizándolos como recelosos, discretos y cerrados. El ser receloso $(\mathrm{N}+)$ se relaciona con otros factores: timidez $(\mathrm{H}-)$ y autosuficiencia (Q2+), rasgos típicos de quienes tienden al aislamiento (tabla 5).

Tabla 5.

Correlaciones, medias y desviaciones estándar para las puntuaciones en los factores de personalidad del 16PF5 en el grupo de infractores frecuentes.

\begin{tabular}{|c|c|c|c|c|c|c|c|c|c|c|c|}
\hline Medida & 16PF-E & $16 \mathrm{PF}-\mathrm{G}$ & $16 \mathrm{PF}-\mathrm{H}$ & 16PF-M & $16 \mathrm{PF}-\mathrm{N}$ & $\begin{array}{c}\text { 16PF- } \\
\text { Q2 }\end{array}$ & $\begin{array}{c}\text { 16PF- } \\
\text { Q4 }\end{array}$ & MI & EXT & $M$ & $\mathrm{DE}$ \\
\hline 16PF-C & ,086 &, $238^{* *}$ &, $424^{* * *}$ &,$- 500^{* * *}$ & $-182^{*}$ &,$- 264^{* * *}$ &,$- 540^{* * *}$ &, $567^{* * *}$ & , 049 & 4,37 & 1,62 \\
\hline 16PF-E & & 117 & 144 &,- 107 & ,075 &,- 003 & ,063 &,- 027 & ,063 & 4,79 & 1,45 \\
\hline 16PF-G & & & ,263*** &,$- 448^{* * *}$ & ,037 &,- 096 &,$- 249 * *$ &, $410^{* * *}$ & ,171* & 4,93 & 1,60 \\
\hline 16PF-H & & & &,$- 363^{* * *}$ &,$- 309^{* * *}$ &,$- 421^{* * *}$ &,$- 276^{* * *}$ &, $392^{* * *}$ &, $468^{* * *}$ & 4,77 & 1,68 \\
\hline 16PF-M & & & & & ,239** & $327^{* * *}$ &, $401^{* * *}$ &,$- 577^{* * *}$ &,$- 200^{*}$ & 6,97 & 1,52 \\
\hline 16PF-N & & & & & &, $568^{* * *}$ & ,204** &,- 143 &,$- 570^{* * *}$ & 6,11 & 1,47 \\
\hline 16PF-Q2 & & & & & & & $270^{* * *}$ &,$- 270^{* * *}$ &,$- 715^{* * *}$ & 7,09 & 1,57 \\
\hline 16PF-Q4 & & & & & & & &,$- 465^{* * *}$ &,- 081 & 6,94 & 1,49 \\
\hline MI & & & & & & & & & ,054 & 4,51 & 1,80 \\
\hline EXT & & & & & & & & & & 4,33 & 1,83 \\
\hline
\end{tabular}

${ }^{*} p \leq 0,05^{* *} p \leq 0,01{ }^{* * *} p \leq 0,001$. 
El $62,7 \%$ de los infractores frecuentes se ubicó en el polo alto del factor Q2, autosuficiencia (tabla 3), con una diferencia significativa en la media del grupo respecto a los no infractores frecuentes (tabla 4), indicando que los transgresores habituales tienden a sentirse más seguros, solitarios e individualistas. A mayor puntuación en Q2, más tendencia a la soledad, dificultad para actuar cómodamente con otros por considerarlos lentos o ineficaces. La tabla 5 muestra la correlación más alta entre Q2 (+) y EX (-) en el grupo infractores frecuentes, indicando en ellos independencia y aversión a la colectividad.

La tabla 3 muestra que el $56,5 \%$ de los infractores frecuentes se halló en el polo alto del factor Q4 (tensión), con una diferencia significativa con la media del grupo control (tabla 4), indicando que tienen una mayor tendencia a ser tensos, enérgicos, impacientes y a sentirse presionados. Este rasgo está asociado a ansiedad flotante y frustraciones generalizadas. La tensión puede conducir a comportamientos afanosos y hábitos nerviosos, conductas de escasa tolerancia a la frustración, impaciencia e irritabilidad, perjudicando la eficiencia del funcionamiento de la persona en las tareas de conducción. Q4 y C se correlacionan (tabla 5) porque la estabilidad emocional es proporcional a la serenidad para enfrentar las exigencias sociales.

El 30,5\% de los infractores frecuentes se ubicó en el polo bajo del factor manipulación de imagen (tabla 3), evidenciando poco interés por dar una buena impresión y problemas en la autoestima. La media del grupo fue significativamente más baja que la del grupo control (tabla 4). Las puntuaciones bajas son indicadoras de indiferencia o desprecio por las opiniones de los demás, institución o autoridad. Predisposición a rechazar conductas socialmente deseables.

El 33,1 \% de los infractores frecuentes puntuó bajo en el factor extraversión (tabla 3), obteniendo una media significativamente inferior a la del grupo control (tabla 4). Estas bajas puntuaciones señalan tendencia a la introversión e inhibición social, propensión a estar preocupado, posible retraimiento patológico, indicadores de conflictos en el ajuste general, síntomas relacionados con la agresión, dificultades para diferir las necesidades y comportarse teniendo en cuenta las consecuencias a largo plazo.

El análisis estadístico de las escalas: factor A (afabilidad), factor B (razonamiento) y factor I (sensibilidad), no mostró diferencias significativas en las medias de ambos grupos (tabla 4), pero en los porcentajes de sujetos ubicados en polos extremos sí se evidencian tendencias (tabla 3). El que la exploración estadística no logre mostrar una diferencia significativa puede explicarse por el alto porcentaje de puntuaciones neutras y la tendencia a compensar los valores extremos.

Las bajas puntuaciones obtenidas por el grupo infractores frecuentes en los factores afabilidad (A) y estabilidad emocional ( $C$ ), muestran que tienen dificultades para interactuar de manera efectiva con otros. Por lo general, el bajo grado de afabilidad afecta la asertividad, aspecto coincidente con la correlación significativa entre el factor A y el $\mathrm{H}$ en el grupo infractores frecuentes (tabla 5). La combinación de puntuaciones muy bajas en afabilidad (A-) y privacidad ( $\mathrm{N}$-), en este grupo, indica confusión en el modo de relacionarse con los demás. Las puntuaciones bajas en afabilidad (A-) unidas a los altos valores en autosuficiencia (Q2+), señalan que los infractores habituales tienden a la independencia y a ser toscos en el trato con los demás.

Los valores obtenidos por los infractores frecuentes en la escala de razonamiento (Factor B) indican tendencia a tener pensamiento concreto, pese a que el $18,2 \%$ son profesionales (tabla 3 ). La media del conjunto es menor que en el grupo control (tabla 4). Altos puntajes en el factor B, se asocian con la capacidad de anticipación de contingencias, control de impulsos, y resolución de problemas. Los bajos puntajes en el grupo infractor frecuente en este factor, pueden ayudar a explicar la reincidencia en sus infracciones de tránsito.

Finalmente, se realizó un análisis de regresión logística binaria, mediante el método INTRO con aquellos factores que evidenciaron diferencias significativas entre los dos grupos de conductores, 
con el fin de identificar las variables independientes que tienen un mayor peso en la asociación y capacidad de predicción.

En la tabla 6, las variables que asocian significativamente con las infracciones habituales a las normas de tránsito son el sexo, la dominancia y la privacidad. Ser varón, tener menos dominio de la ira, poca asertividad y mayor tendencia a ser re- celoso y encerrado en sí mismo, pronostican el ser infractor frecuente de tránsito. Las variables explican el $14,9 \%$ de la varianza de las infracciones habituales. Los hombres tienen 2,17 oportunidades más que las mujeres de ser infractores frecuentes de tránsito. A menor dominancia, la posibilidad de ser infractor frecuente aumenta en 0,78; y a mayor recelo y encierro en sí mismo, la oportunidad de ser infractor frecuente crece en 1,29.

Tabla 6.

Resumen del análisis de regresión logística que pronostica infracciones frecuentes de tránsito.

\begin{tabular}{lcccc}
\hline & B & EE & $\begin{array}{c}\text { Cociente de } \\
\text { probabilidad }\end{array}$ & $\begin{array}{c}\text { Estadística } \\
\text { de Wald }\end{array}$ \\
\hline Sexo &, 776 &, 312 & 2,173 & $6,200^{*}$ \\
\hline Edad &, 387 &, 277 & 1,472 & 1,954 \\
\hline Factores de Personalidad & & & & \\
\hline C - Estabilidad Emocional &,- 023 &, 108 &, 978 &, 044 \\
\hline E - Dominancia &,- 248 &, 098 &, 781 & $6,381^{*}$ \\
\hline G - Atención a las normas &,- 089 &, 091 &, 915 &, 965 \\
\hline H - Atrevimiento &, 019 &, 096 & 1,019 &, 040 \\
\hline M - Abstracción &, 069 &, 112 & 1,071 &, 376 \\
\hline N - Privacidad &, 251 &, 110 & 1,286 & $5,245^{*}$ \\
\hline Q2 - Autosuficiencia &, 019 &, 129 & 1,019 &, 021 \\
\hline Q4 - Tensión &, 083 &, 104 & 1,086 &, 627 \\
\hline MI - Manipulación de imagen &,- 015 &, 098 &, 985 &, 023 \\
\hline EXT - Extraversión &, 053 &, 118 & 1,055 &, 205 \\
\hline Actitud & & & & \\
\hline Creencias &,- 323 &, 242 &, 724 & 1,790 \\
\hline Comportamientos &, 153 &, 258 & 1,165 &, 351 \\
\hline Conocimientos &,- 009 &, 078 &, 991 &, 013 \\
\hline
\end{tabular}

${ }^{*} \mathrm{p} \leq .05,{ }^{* *} \mathrm{p} \leq .01$.

\section{Discusión}

En consonancia con Montoro, Alonso Plá y cols. (2003), este estudio ratifica la convicción de que el factor humano sigue siendo uno de los aspectos más complejos en la consideración de los componentes de la seguridad vial. La conducción temeraria, la falta de respeto a las normas de tránsito y las imprudencias al conducir están íntimamente relacionados con las características de persona- lidad de los conductores. Más investigaciones en esta área siguen siendo necesarias para fundamentar mejor las campañas de educación en movilidad y seguridad vial.

En esta investigación el objetivo de establecer el perfil psicológico de conductores infractores frecuentes de normas de tránsito permitió encontrar diferencias significativas en algunos factores de 
personalidad, según la comisión de infracciones habituales o no de normas de tránsito en conductores multados. Esta correspondencia hallada, coincide con algunos resultados de las investigaciones de Taubman-Ben-Ari, Mikulincer y Gillath (2004) sobre estilos de conducción y rasgos de personalidad, específicamente en lo que respecta al factor ansiedad.

Existe también coincidencia con las conclusiones del segundo estudio de Taubman-Ben-Ari y cols. (2012), en relación a la correspondencia entre estilo de pensamiento y modos de conducción, referido en nuestros resultados a los nexos entre creencias y comportamientos.

En lo que respecta a las diferencias en las variables de género y edad, así como en el estudio de Taubman-Ben-Ari y cols. (2004) encontramos que los hombres presentan una mayor propensión a las infracciones frecuentes de normas de tránsito que las mujeres, y que los varones son más proclives a tener conducción riesgosa y mayor riesgo de accidentes que las mujeres. También se coincide con Aniței, Chraif, Burtăverde y Mihăilă (2014), respecto a que la inestabilidad emocional y el género masculino son probables predictores de infracciones de normas de tránsito y conducción riesgosa, mientras las mujeres tienden a tener más estabilidad emocional, estrés y afabilidad que los hombres, estos se orientan hacia el disfrute de la violencia, a la actuación agresiva y al negativismo en la conducción frente a las normas de tránsito.

En la variable edad se evidenciaron contrastes en los resultados de nuestra investigación y los de Taubman-Ben-Ari y cols. (2010), mientras que para estos autores los jóvenes varones conductores (18-25 años) son más propensos a la conducción agresiva y temeraria, en nuestro estudio los hombres infractores frecuentes de las normas de tránsito se ubican entre los 36 y 50 años de edad.

El alto puntaje en el factor M (abstracción e impracticabilidad, y ensimismamiento), obtenido por la muestra de infractores frecuentes de normas de tránsito, coincide con las conclusiones del trabajo de Ledesma, Montes, Poó y López (2009), Montes, Poó, Valle y López $(2012,2014)$, que in- dican la relación entre personalidad disociativa, errores atencionales en la conducción e incidentes de tránsito.

Por otro lado, los resultados en relación a la variable nivel educativo de la población evaluada contradicen los hallazgos de Norza, Granados, Useche, Romero y Moreno (2014), para quienes los conductores con estudios superiores alcanzados inciden en menos accidentes; contrario a esto, la presente investigación evidenció que los infractores frecuentes de normas de tránsito, poseen nivel educativo con estudios de bachillerato, técnica, tecnológico, pregrado y posgrado. No obstante, en las variables de género y factores de personalidad, ambos estudios presentan significativas coincidencias.

\section{Referencias}

Alonso, F., Esteban, C., Calatayud, C., Medina, J., Montoro, L. y Egido, Á. (2003). Formación y Educación Vial. Attitudes. Cuadernos de reflexión. Instituto Universitario de Tráfico y Seguridad Vial (INTRAS). España. Recuperado de http:// www.attitudes.org/wp-content/download/ cuadernos_de_reflexion/03_Formacion_y_ educacion_vial.\%20Una_vision_a_partir_de_ algunas_practicas_internacionales.pdf

Aniței, M. Chraif, M., Burtăverde, V. \& Mihăilă, T. (2014). The Big Five personality factors in the prediction of aggressive driving behavior among romanian youngsters. International Journal of Traffic and Transportation Psychology 2(1), 7-20. Recuperado de http://www. ijttp.ro/files/vol2no1/2art2.pdf

Bernstein, E. M. \& Putnam, F. W. (1986). Development, reliability, and validity of a dissociation scale. Journal of Nervous and Mental Disease, 174, 727-735, doi: 10.1097/00005053198612000-00004.

Caprara, G. V., Barbaranelli, C. \& Borgogni, L. (1993). Big Five questionnaire. (Trad. cast. Bermúdez et al. Madrid; TEA, 1995). Florencia: Organización speciali. 
Cattell, R., Cattell, A. \& Cattell, H. (1993). Sixteen personality factor questionnaire. Fifth edition. (Adaptación española Seis dedos, N. TEA, ediciones, 2013). Champaign, IL: Institute for Personality and Ability Testing, Inc.

Corporación Fondo de Prevención Vial. (2010). Primer estudio de comportamiento y seguridad vial. Recuperado de http://www.fpv. org.co/uploads/repositorio/1erestudiode_ comportamiento_y_seguridad_vial.pdf

Corporación Fondo de Prevención Vial. (2011). Anuario estadístico de accidentalidad vial Colombia. Recuperado de http://www.fpv. org.co/uploads/documentos /libreria/anuario_2011_pagina.pdf

Corporación Fondo de Prevención Vial. (2012). Informes generales de accidentalidad y exposición 2007-2012. Recuperado de http://www. fpv.org.co/images/ repositorioftp/2\%20Antioquia\%20nov\%2014-13.pdf

Elander, J., West, R. \& French, D. (1993). Behavioral correlates of individual differences in roadtraffic crash risk: Anexamination of methods and findings. Psychological Bulletin, 113, 279294, doi: 10.1037/0033-2909.113.2.279.

Hansen, C. P. (2005). Personality characteristics of the accident involved employee. Journal of Business \& Psychology, 2(4), 346-365, doi: 10.1007/BF01013766.

Jamenson, R. E., McLellan, A. T. \& Jackson, T. B. (1971). Perceptual style and traffic accidents. Harrisburg, PA: Governor's council on drug and alcohol abuse.

Knapper, C. \& Cropley, A. (1981). Social and interpersonal factors in driving. Rev. Progress in Applied Social Psychology, 1, 191-220, doi: 10.1111/j.1464-0597.1980.tb01104.x.

Karson, M., Karson, S. \& O’Dell, J. (2011). 16PF-5. Una guía para su interpretación en la práctica clínica. España: Tea Ediciones, sexta edición.

Ministerio de Transporte. República de Colombia. (2002). Código Nacional de Tránsito. Ley 769 de 2002. Recuperado de https://www.mintransporte.gov.co/documentos.php?id=17

Norza, C. E., Granados, L. E., Useche, H. S., Romero, H. M. \& Moreno R. J. (2014). Componentes descriptivos y explicativos de la accidentalidad vial en Colombia: incidencia del factor humano. Revista Criminalidad, 56(1), 157-187.

Ledesma, R., Montes, S., Poó, F. y López-Ramón, M. (2010). Las diferencias individuales en la falta de atención del conductor: los errores de conducción de atención relacionados con escala. Traffic Injury Prevention, 11(2), 142150, doi: 10.1080/15389580903497139.

Ministerio de Transporte. República de Colombia. (2010). Manual de infracciones. Recuperado de http://www.policia.gov.co/portal/page/ portal/Direccion_Transito_transportes/Documentacion/Tab/MANUAL\%20DE\%20INFRACCIONES_0.pdf

Montero, I. \& León, O. G. (2007). A guide fornaming research studies in psychology. International Journal of Clinical and Health Psychology, 7(3), 847-862.

Montes, S., Poó, F., Valle, V. y López, S. (2012). Tendencias disociativas e incidentes de tránsito. Revista Argentina de Ciencias del Comportamiento, 4(1), 4-10. Recuperado de http://www.redalyc.org/articulo.oa?id= 333427356002

Montes, S, Ledesma, R. y Poó, F. (2014). Estudio y prevención de la distracción e inatención en la conducción. Avances en Psicología Latinoamericana, 32(1), 115-129, doi: http://dx.doi. org/10.12804/apl32.1.2014.08

Parker, D., Manstead, A., Stradling, S. \& Reason, $\mathrm{J}$ (1992). Determinats of intention to commit driving violations. Rev. Accident Analysis and Prevention, 24, 117-131, doi: 10.1016/00014575(92)90028-H.

Ponce, C. (2015). Dimensiones sintomáticas psicopatológicas en conductores de Lima metropolitana. Rev. LIBERABIT, 21(1), 153-165. 
Lima, Perú. Recuperado de http://revistaliberabit.com/es/revistas/liberabit21_1/13_ vera.pdf

Reason, J. T., Manstead, A., Stradling, S., Baxter, J. \& Campbell, K. (1990). Errors and violations on the road: A real distinction? Rev. Ergonomics, 33(10-11), 1315-1332, doi: 10.1080/00140139008925335.

Secretaría de Movilidad. Alcaldía de Medellín. (2014). Informe anual de accidentalidad, 2014. Recuperado de https://www.medellin. gov.co/movilidad/

Summala, H. \& Näätänen, R. (1974, december). A model for the role of motivation all factors in drivers decision - making. Rev. Accident Analysis \& Prevention, 6(3), 243-261, doi: 10.1016/0001-4575(74)90003-7.

Sümer, N. (2003, november). Personality and behavioral predictors of traffic accidents: testing a contextual mediated model. Rev. Accident Analysis \& Prevention, 35(6), 94964, doi: 10.1016/S0001-4575(02)00103-3.

Stokols, D., Novaco, R. \& Stokols (1978). Traffic congestion, Type a behavior, and stress. Jour- nal of Applied Psychology, 63(4), 467-480, doi.:10.1037/0021-9010.63.4.467.

Taubman-Ben-Ari, O., Mikulincer M. \& Gillath O. (2004, may). The multidimensional driving style inventory-scale construct and validation. Rev. Accident Analysis \& Prevention, 36(3), 323-332, doi: 10.1016/S0001-4575(03)00010-1.

Taubman-Ben-Ari, O. \& Yehiel D. (2012, march). Driving styles and their associations with personality and motivation. Accident Analysis \& Prevention, 45, 416-422, doi: 10.1016/j. aap.2011.08.007.

Vargas, D. A. (2015, julio). Comportamiento de muertes y lesiones por accidentes de transporte. Colombia 2014. Forensis, 16(1), 351-406. Recuperado de http://www.medicinalegal. gov.co/documents/88730/1656998/Forensis+ Interactivo+2014.24-JULpdf.pdf/9085ad79d2a9-4c0d-a17b-f845ab96534b

Wilde, G. (1982, december). The theory of risk homeostasis: Implications for safety and health. Rev. Risk Analysis, 2(4), 209-255, doi: 10.1111/ j.1539-6924.1982.tb01384. 